\title{
Longitudinal integrated clerkships: to educate what remains after one has forgotten what one has learned in the classroom
}

\section{Ki Jun Han}

\section{Department of Internal Medicine, International St. Mary's Hospital, Catholic Kwandong University College of Medicine, Incheon, Korea}

Albert Einstein once said, "Education is what remains after one has forgotten what one has learned in school." I believe this sentence accurately expresses the direction competency-based education should take. If that happens, as part of a clinical clerkship curriculum, what are the competencies needed to become a good doctor that cannot be taught in classrooms but which one must possess? How can they be taught?

Catholic Kwandong University College of Medicine attempted to create practical and effective curriculums to foster good doctors by encompassing the advantages of integrated medical curriculum and the meaning of medical humanities in the outcome-based education system, when the education curriculums were fully reformed in 2014. While implementing this, a need emerged to provide more integrated curriculums that could impart the competencies deemed unattainable under the existing curriculums of our university. As a result, the following was introduced in the curriculums in 2017, according to the trend of global medical education: humanistic approach for patient safety, early clinical experience, going beyond hospital toward society, and student-driven learning based on diverse methods. In this context, longitudinal integrated clerkships (LIC) were developed in accordance with the education philosophy and situation of our university, and were introduced in 2019 as a part of the clinical clerkships targeting third-year medical school students.

The clinical clerkships at Catholic Kwandong University College of Medicine were conducted along the lines of a rotation block clerkship of a large tertiary general hospital, similar to any other medical school in South Korea. A decision was taken to implement the LIC curriculum with the goal of providing continual and responsible patient experiences, which were the basic attributes of medical care, to students, while overcoming the limitations of such an educational environment: for example, fragmented and subdivided medical treatment patterns, restricted continual experience of core diseases or patients of minor severity level, and negative influence on adopting a patient-oriented treatment attitude [1]. Targeting all third-year medical school
Received: August 4, 2019 • Revised: August 7, 2019 • Accepted: August 7, 2019 Corresponding Author: Ki Jun Han (https://orcid.org/0000-0003-3398-6948) Department of Internal Medicine, International St. Mary's Hospital, Catholic Kwandong University College of Medicine, 25 Simgok-ro 100beon-gil, Seo-gu, Incheon 22711, Korea Tel: +82.32.290.3793 Fax: +82.32.290.3879 email: daniel@cku.ac.kr
Korean J Med Educ 2019 Sep; 31(3): 277-279.

https://doi.org/10.3946/kjme.2019.138

eISSN: 2005-7288

(C) The Korean Society of Medical Education. All rights reserved. This is an open-access article distributed under the terms of the Creative Commons Attribution Non-Commercial License (http:// creativecommons.org/licenses/by-nc/3.0/), which permits unrestricted non-commercial use, distribution, and reproduction in any medium, provided the original work is properly cited. 
students, our university conducted LIC of the amalgative clerkship type while maintaining the conventional rotation block clerkship.

Do you empathize with the education philosophy and goal of LIC? Just as social agreement is required when a certain policy is enforced, agreement between teaching faculties is required on the basis of empathy when a new curriculum is implemented. The resistance of internal members was strong during the introduction of LIC. Although they agreed with the goal of LIC, they thought that more preparation and time were needed before starting a completely new form of educational program as regular curriculum instead of elective clerkship. At that time, someone said, "Let's say there are two types of pictures here, a blueprint and a landscape painting. On the blueprint, the first line must be drawn carefully; otherwise the blueprint will not be accurate. However, when you paint a landscape, you don't have to be so careful when drawing the first line. Once you have drawn the first line, you will finish the painting gradually as you paint continually. There is no way of knowing where the first line is in the finished landscape painting, and you don't have to know. Education is closer to the latter. If we agree on the goal of LIC, let's draw a line first."

We thought over why LIC were needed in our education curriculum. Based on our conclusions, we developed an education philosophy and created our university's own educational program to implement that philosophy.

First, a "longitudinal” program was developed. "Continuity" has been implemented, which is the core concept of LIC-continuity of student-patient meeting, of student-supervising professor relationship, of the given educational environment, and of mutual teaching between student colleagues [2].

For the continuity of student-patient meeting, the clinical department assigned a patient panel to each student to let him/her experience the patients longitudinally, and the student performed longitudinal observation on his/her own. Furthermore, volunteers who narrated their illness experience to the medical school students were accepted among the patients coming from a retirement village close to the university hospital, and they participated in another group of patient panel.

The continuity of student-supervising professor relationship and of the given educational environment are concepts of clerkship, and to implement the continuity of mutual teaching between student colleagues, studentoriented seminars were held. As such, efforts were made to implement patient-oriented integrated medical care in accordance with the current situation of our university while maintaining the continuity.

Second, seven student-oriented seminars were held to educate about "integrated" patient medical care. For the seminar contents, integrated educational programs (team approach, medical ethics, caring/palliative care, clinical decision-making, and so forth) developed by the Medical Education Center, Seoul National University College of Medicine, were shared through the LIC consortium [3]. Before proceeding with the main seminar, it was determined that humanities curriculums were required to develop the need to learn the method of integrated patient treatment in the minds of students. The medical humanities seminar was held 4 times, so that students could develop their own thoughts on doctor-patient relationship while discussing "bedside manners" by Abraham Verghese and "narrative medicine" by Rita Charon. Yearning for a doctor engaging in clinical reasoning was seeded, and they thought about why physical examination was important even in the current medical environment of cutting-edge devices. The students agreed with the following: physical examination is important for making a decision regarding which 
examination device to be used and has a power that changes the perception of person-person relationship to that of doctor-patient relationship: that is, relationship between a patient suffering from a disease and a doctor trying to help that person. Furthermore, an opportunity was given to think that the ability to recognize, understand, sympathize, and absorb an illness experience felt by a patient rather than by a doctor was an emotional competency that doctors of the artificial intelligence age needed to have.

Third, an effort was made to create an educational environment to implement the traditional concept of clinical clerkship. The meaning of clerkship is well captured in a saying by Benjamin Franklin: "Tell me and I forget, teach me and I may remember, involve me and I learn." During the 20 weeks of LIC, the students participated as members of a medical team in outpatient care and did rounds in the clinical department, where they were assigned on a Monday afternoon every other week.

Although we did not have a perfect plan, we drew the first line. One semester has passed, and the first LIC session of 20 weeks has finished. There were many trials and errors. Because it is too early to conduct a systematic evaluation, only feedback was taken from the students through a survey. Feedback from one student shows a picture in advance, which we are going to paint continually and finish.

"Thanks to the LIC, I had a chance to think deeply about the quality of life of chronic patients. I was given the chance to think over the relationships of person and person, doctor and patient, and disease and person. It was an opportunity for me to open my eyes, at least a little, to the life I am going to live as a doctor and the attitude I am going to adopt."

\section{ORCID:}

Ki Jun Han: https://orcid.org/0000-0003-3398-6948

Acknowledgements: The author would like to express gratitude to the LIC consortium of Korea Association of Medical Colleges for providing consultation on the implementation process of Catholic Kwandong University LIC program.

Funding: None.

Conflicts of interest: No potential conflict of interest relevant to this article was reported.

Author contributions: All work was done by Ki Jun Han.

\section{References}

1. Lee YM. The concept and background for introducing longitudinal integrated clerkships. Korean Med Educ Rev. 2018;20(1):1-5.

2. Yeo S. Development and operation of longitudinal integrated clerkship programs in overseas medical schools. Korean Med Educ Rev. 2018;20(1):6-14.

3. Korea Association of Medical Colleges. Present and future of academic medicine in Korea. Seoul, Korea: Korea Association of Medical Colleges; 2017. 